\title{
Current Concepts and New Trends in the Treatment of Cardiogenic Shock Complicating Acute Myocardial Infarction
}

\author{
Theodora Benedek*, Dan Dobreanu \\ University of Medicine and Pharmacy of Tirgu Mures, 38 Gheorghe Marinescu street Tirgu Mures, 540139, Romania
}

\begin{abstract}
Cardiogenic shock (CS) is a critical condition which often complicates the evolution of an acute myocardial infarction (AMI). At the same time, co-existence of chronic multi-vessel disease can lead to the development of cardiogenic shock in cases with pronounced haemodynamic instability. Different clinical studies have tried to identify the most appropriate treatment for critical cases of CS complicating AMI. This review aims to present the current status of recommended therapeutic strategies for severe cases of CS presenting as a complication of AMI, and try to shed light on the most appropriate therapeutic strategy as outlined in the current literature. The paper will discuss the different current strategies available for use in the treatment of this condition, includig interventional revascularisation, (complete or culprit), the role of new devices for providing mechanical circulatory support, and the potential role of new drug therapies and of hypothermia.
\end{abstract}

Keywords: cardiogenic shock, myocardial infarction, revascularisation

Received: 05 January 2015 / Accepted: 15 January 2015

Cardiogenic shock (CS) is a critical condition which often complicates the evolution of an acute myocardial infarction (AMI). Large infarcts are frequently associated with a severe reduction in cardiac output, especially in occlusions of the Left Main (LM) or Left Anterior Descending Artery (LAD). These arteries supply the anterior cardiac wall, which is the most important contributor to left ventricular global performance, and their occlusion has a significant impact on over-all ventricular function [1]. CS usually develops when more than $40 \%$ of the total ventricular myocardial mass is akinetic, as a consequence of the infarction, and this happens usually in the context of a large anterior infarction [2]. However, right ventricular (RV) infarction may also be associated with CS, though predominant $\mathrm{RV}$ shock is rare [3]. At the same time, coexistence of a chronic multi-vessel disease may favour the development of cardiogenic shock in cases with pronounced haemodynamic instability [4].
Cardiogenic shock frequently occurs in the acute stage of an infarction and raises difficult questions regarding the optimal therapeutic strategy to be used to reopening the occluded coronary artery. Despite recent progress in the treatment of myocardial infarction, the mortality rate remains high at $50 \%$ in CS associated with AMI. Moreover $50 \%$ of deaths occur in the first 48 hours after the patient has been admitted to hospital [5]. This results from an advancement of severe pump failure, despite the administration of modern therapeutic measures including revascularisation, intraaortic balloon counterpulsation or usage of circulatory support devices.

Different clinical studies have tried to identify the most appropriate treatment for critical cases of CS complicating AMI. This review aims to present the current status of recommended therapeutic strategies in the light of the recent advancements in the treatment of this critical condition.

\footnotetext{
* Correspondence to: Prof. Theodora Benedek, MD, PhD, University of Medicine and Pharmacy of Tirgu Mures, 38 Gheorghe Marinescu street, Tirgu Mures, 540139, Romania; e-mail: theodora.benedek@umftgm.ro
} 
Current questions related to selection of the most appropriate therapeutic strategy in CS complicating AMI are summarised in Table 1.

\section{CONSERVATIVE TREATMENT OR REVASCULARISATION?}

The first landmark trial attempting to answer the question of whether conservative treatment or revascularisation was better, was the SHOCK trial (SHould we use emergently revascularize Occluded Coronaries in cardiogenic shocK? NCT00000552), which showed that although overall mortality at 30 days was not significantly reduced by revascularization compared with the conservative group ( $46.7 \%$ vs $56.0 \%, \mathrm{p}=0.11$ ), sixmonth mortality was significantly lower in the revascularization group ( $50.3 \%$ vs $63.1 \%, p=0.027)$. The study concluded that early revascularization is the procedure of choice for patients with acute myocardial infarction complicated by cardiogenic shock [6-12].

In a Natiowide Inpatients Sample Study, analysis of data from 60,833 patients with AMI and CS indicated that a revascularisation strategy is associated with a significantly lower mortality compared with a conservative strategy, in all patients groups, including the elderly [13]. The results of this study suggested a clear need for a more aggresive management approach in case of CS complicating AMI.

Timing of primary percutaneous intervention (PCI) is another key issue in CS. Although it is generally accepted that primary PCI should only be performed in the first 12 hours after the onset of symptoms in AMI, in the case of CS complicating myocardial infarction, a survival benefit may be recorded even when PCI is performed at 48 hours after the onset of ischemia and at 18 hours after the onset of shock $[4,14]$.

\section{- COMPLETE REVASCULARISATION OR CULPRIT VESSEL REVASCULARISATION?}

Up to $87 \%$ of patients with CS complicating AMI have a multi-vessel disease. The strategy of multi-vessel revascularization employs balloon angioplasty and stenting of all the lesions in both infarct-related artery (defined as culprit lesion revascularization) and non-infarct related arteries, which have been identified by a coronary angiography, performed in the acute phase [15]. As myocardial infarction is caused by a sudden occlusion of the coronary flow in one of the three coronary arteries, while in many cases severe coronary stenosis coexist in the other two coronary arteries, or even in the same infarct-related artery, but proximal or distal to the culprit lesion, one logical question is whether all the lesions identified during coronary angiography should be stented in the emergent condition or only the one responsible for infarction.

In stable conditions, multi-vessel revascularization leads to unnecessary prolongation of intervention times and complications, therefore the so called "staged" intervention is planned, represented by immediate revascularization of only the culprit lesion (i.e. the coronary plaque whose abrupt occlusion caused the infarction), followed by stenting of the other lesions at a later stage, when the myocardium has recovered its function and metabolism. It should be noted, that it is far from easy to identify the culprit lesion, especially in cases with non-obstructive coronary plaques. In these case, imag-

Table 1. Crucial questions in treatment of patients with CS and AMI 
istic techniques may be crucial in the identification of myocardial areas with akinesia (marker of infarction), by echocardiography, or the exact location of infarction by modern magnetic resonance techniques or positron emission tomography, correlated with the distribution of the coronary arteries supplying the territory suffering from ischemic injury.

On the other hand, in cases of CS complicating an AMI, the haemodynamic instability may require a more aggressive approach consisting in complete revascularization of all significant coronary artery stenoses, as the revascularization of the culprit lesion alone may be insufficient in re-establishing the haemodynamic balance of a severely affected myocardium [16]

The ongoing CULPRIT-SHOCK trial (Prospective Randomized Multicentre Study Comparing Immediate Multi-vessel Revascularization by PCI Versus Culprit Lesion PCI With Staged Non-culprit Lesion Revascularization in Patients With Acute Myocardial Infarction Complicated by Cardiogenic Shock. NCT01927549) tries to identify if multi-vessel revascularization and stenting was more efficient than the revascularization of the culprit vessel alone, possibly followed, in patients with cardiogenic shock and acute myocardial infarction, by stent implantation in the other vessels at a later stage.

In this trial, two approaches will be compared:

a. Immediate multi-vessel PCI, represented by stenting of the culprit vessel followed by immediate stenting of all additional stenoses higher than $70 \%$ in any other major coronary artery,

b. Stenting only the culprit lesion, leaving all other lesions untreated in the acute phase, though subsequently they will be re-vascularized if non-invasive tests identify associated severe ischaemia.

\section{USE OF INTRAAORTIC}

\section{BALLOON COUNTERPULSATION \\ PUMP}

The use of an intra-aortic balloon couterpulsation pump (IABP) remains a controversial issue in the treatment of CS associated with AMI, since the IABPSHOCK II trial (Randomized Clinical Study of Intraaortic Balloon Pump Use in Cardiogenic Shock Complicating Acute Myocardial Infarction. NCT00491036) showed no mortality benefit in patients with CS complicating AMI who underwent early revascularisation
[17]. In this trial, 598 critically ill patients with CS and AMI were randomised to primary angioplasty plus IABP plus standard medical treatment (300 patients) or to primary angioplasty plus medical treatment without IABP (298 patients) [18]. Mortality rates at 30 days were $39.7 \%$ in the IABP group and $41.3 \%$ in no-IABP group, there being no statistically significant difference between the groups $(\mathrm{p}=0.69)$.

However, insertion of an intra-aortic balloon counterpulsation pump was shown to improve coronary perfusion via diastolic inflation and may contribute to afterload reduction via systolic deflation, therefore being useful in certain clinical circumstances. At the same time, in the IABP Cardiogenic Shock Trial (NCT00469248) [19] insertion of IABP led to a significant improvement of cardiac output, increasing from $4.8 \pm 0.5$ to $6.0 \pm 0.5 \mathrm{~L} / \mathrm{min}$, in parallel with a reduction of systemic vascular resistance from $926 \pm 73$ to $769 \pm$ 101 dyn $\cdot \mathrm{s}(-1) \cdot \mathrm{cm}(-5)$.

\section{USE OF NEW DEVICES FOR MECHANICAL CIRCULATORY SUPPORT}

Different new devices have been proposed as adjunctive treatment in order to sustain cardiac output until the recovery of ventricular function following revascularisation or as a bridge to surgical bypass [20].

Three new commercially available devices exist on the market for mechanical circulatory support. The Impella (Abiomed-Impella CardioSystems $\mathrm{GmbH}$, Aachen, Germany), Tandem Heart (Cardiac Assist, Inc, Pittsburgh, Pensylvania) and Extracorporeal Membrane Oxygenation (ECMO) systems are designed to increase cardiac outout, blood pressure and coronary perfusion in parallel with a reduction of pulmonary capillary wedge pressure.

The ISAR-SHOCK study (Efficacy Study of LV Assist Device to Treat Patients with Cardiogenic Shock. NCT00417378) evaluated the safety and efficacy of a left ventricular assist device in comparison to an intraaortic balloon pump (IABP) in patients with cardiogenic shock complicating an acute myocardial infarction [21]. The study showed that LV assist device was superior to IABP in terms of efficacy of haemodynamic support in patients with CS. The left ventricular assist device consists of a small rotary blood pump (Impella LP2.5, Abiomed-Impella CardioSystems $\mathrm{GmbH}$, 
Aachen, Germany), that is placed retrograde through the aortic valve, aspirates the blood from the left ventricle and ejects it to the ascending aorta [22].

The TandemHeart (Cardiac Assist, Inc, Pittsburgh, $\mathrm{Pa}$ ) is another device intended for improving ventricular function, consisting of a cannula inserted into the left atrium via the femoral vein and trans-septal puncture so as to remove and recirculate blood from the left atrium [23].

Extracorporeal membrane oxygenation consist of an implanted veno-arterial system dedicated to increase tissue perfusion. This has the advantage of rapid insertion which is extremely useful in cases with very poor haemodynamic status. The ExtraCorporeal Membrane Oxygenation in the Therapy of Cardiogenic Shockstudy (ECMO-CS.NCT02301819) noted the effect of such a device on ventricular function in patients with severe CS [24].

In the "Comparison of Standard Treatment Versus Standard Treatment Plus Extracorporeal Life Support (ECLS) in Myocardial Infarction Complicated With Cardiogenic Shock" study (NCT00314847), two treatment regimens were compared in 44 patients. One group received standard treatment alone, a second group received standard treatment plus being put on an extracorporeal life support system. The latter consisted of extracorporeal circulation of blood through a membrane oxygenator which was subsequently replaced with an Impella Ventricular Assist device (Impella Cardiosystems AG, Aachen, Germany).

\section{-USE OF NEW DRUGS}

Several new drugs have been suggested in association with already established medical therapy in order to help the recovery of the damaged myocardium or to improve the results of percutaneous revascularization.

For instance, intravenous administration of glycoprotein IIb/IIIa inhibitors during percutaneous interventions has been proved to be safe and effective in patients with CS undergoing a revascularization procedure for an AMI.

A sub-analysis of the PRAGUE-7 study on 37 ventilated patients with CS and AMI revealed that upfront administration of abciximab prior to revascularization led to a significantly lower incidence of the primary composite endpoint of death/stroke/re-infarction/new severe renal failure, which was $53 \%$ in the treatment arm compared with $60 \%$ in the control arm, associated with a significantly better TIMI flow ( 2.75 versus $2.31, \mathrm{p}<0.05)[25,26]$. Another trial that will test this hypothesis is ongoing (Abciximab in Patients Undergoing Percutaneous Coronary Intervention for Cardiogenic Shock trial, NCT00420030), having as endpoints angiographic TIMI flow, left ventricular function and a combined clinical endpoint.

Cyclosporine is another new drug which has been proposed for reduction of re-perfusion injury in cases of large size AMI complicated with CS. The CLOTILDE trial (Cyclosporine in Acute Myocardial Infarction Complicated by Cardiogenic Shock, NCT01901471), tested the hypothesis that administration of cyclosporine during the reperfusion phase of AMI would reduce the infarct size by $20-40 \%$, reduce the risk of multi-organ failure and improve the clinical status of these patients.

In the TRIUMPH study (A Study of the Safety and Efficacy of Nitric Oxide Reduction in Patients With Cardiogenic Shock After a Heart Attack, NCT00112281), Tilarginine Acetate (a drug that prevents the release of nitric oxide) was injected intravenously to test the hypothesis that reduction of nitric oxide (which is released in excess following myocardial infarction) may improve the clinical status and limit further progression of CS by preventing a drop in blood pressure associated with high serum levels of nitric oxide and thus increase the perfusion of vital organs [27].

Pyruvate is a drug which has been proven to contribute to improved cardiac performance. In the study "Effects of Pyruvate in Patients With Cardiogenic Shock and Intra-aortic Balloon Counterpulsation" (NCT00604331), intracoronary administration of pyruvate was shown to be associated with an increase in the cardiac index (CI $2.23 \pm 0.53$ vs. $1.95 \pm 0.45 \mathrm{~L}$ $\min (-1) \mathrm{m}(-2) ; \mathrm{p}<0.05)$ and stroke volume index (SVI, $29 \pm 6$ vs. $26 \pm 5 \mathrm{~mL} \mathrm{~m}(-2) ; \mathrm{p}<0.05)$ [28].

In the LEAF study (Levosimendan in acute heart failure following primary percutaneous coronary intervention-treated acute ST-elevation myocardial infarction), administration of Levosimendan calcium sensitizer currently used for the improvement of ventricular function in the treatment of advanced cases of chronic heart failure, significantly increased myocardial contractility in patients with AMI complicated by CS, as was demonstrated by a superior improvement of wall motion score index (from $1.94 \pm 0.20$ to $1.66 \pm 0.31$ vs. $1.99 \pm 0.22$ to $1.83 \pm 0.26$, respectively, $\mathrm{p}=0.031$ ) [29] 
Available online at: www.jccm.ro

\section{HYPOTHERMIA FOR CS COMPLICATING} AMI

Hypothermia is now an accepted therapeutic strategy in severe cases of CS associated with AMI, especially in those cases which present with cardiac arrest. Different cooling protocols have been proposed for cases with cardiac arrest, however the role of this treatment in the context of CS complicating an AMI, has yet to be evidenced. An ongoing prospective, randomized, single-centre, controlled, open-label pilot-study (NCT01890317) to investigate whether induction of mild hypothermia in addition to primary percutaneous coronary intervention and optimal medical therapy in myocardial infarction complicated by cardiogenic shock, improves cardiac power index after $24 \mathrm{~h}$, tests the hypothesis that invasive cooling for 24 hours associated with primary percutaneous revascularization and optimal medical therapy may have a significant impact on reduction of the infarct size and improvement of clinical outcomes [30].

\section{-TREATMENT OF CS CAUSED BY MECHANI- CAL COMPLICATIONS IN AMI}

Mechanical complications in AMI with a high potential to progress to CS include rupture of the ventricular wall with consequent cardiac tamponade, rupture of the ventricular septum, causing an acute ventricular septal defect, or of the papillary muscle, leading to acute mitral insufficiency. In all of these cases, timely surgical intervention is crucial for survival of these patients and in many, IABP may be used to stimulate haemodynamic stabilization prior to subsequent surgery [31,32].

\section{- CONCLUSIONS}

Despite all the recent progress in the development of different devices and introduction of new interventional techniques, the mortality associated with CS complicating AMI remains high.

Multiple factors should be considered when selecting the best therapeutic choice in these critical cases with many questions still being tested in ongoing clinical trials in the attempt to identify the role of new drugs, devices or strategies.

At the interface of cardiology and intensive care where complex treatment is required for the critically
The Journal of Critical Care Medicine 2015;1(1) • 9

ill patient, the need of close cooperation and a synergistic approach between these two specialities is essential in ensuring the most appropriate treatment.

\section{CONFLICT OF INTEREST}

None declared.

\section{DEFERENCES}

1. Kaminski KA, Tycinska AM, Stepek T, et al. Natural history and risk factors of long-term mortality in acute coronary syndrome patients with cardiogenic shock. Adv Med Sci. 2014;59:156-60.

2. Menon V, White H, LeJemtel T, Webb JG, Sleeper LA, Hochman JS. The clinical profile of patients with suspected cardiogenic shock due to predominant left ventricular failure: a report from the SHOCK Trial Registry. SHould we emergently revascularize Occluded Coronaries in cardiogenic shock? J Am Coll Cardiol. 2000;36(Suppl A):1071-6.

3. Jacobs AK, Leopold JA, Bates E, et al. Cardiogenic shock caused by right ventricular infarction. A report from the SHOCK registry. J Am Coll Cardiol. 2003;41:1273-9.

4. Reynolds HR, Hochman JS. Cardiogenic Shock: Current Concepts and Improving Outcomes. Circulation. 2008;117:686-97.

5. Tchantchaleishcili V, Schubmehl H, Swartz MF, Hallinan W, Massey HT. Evolving strategies in the treatment of acute myocardial infarction-induced cardiogenic schock. Am Cardiothorac Surg. 2014;3:606-11.

6. Hochman JS, Sleeper LA, Webb JG, et al. Early revascularization in acute myocardial infarction complicated by cardiogenic shock. SHOCK Investigators. Should We Emergently Revascularize Occluded Coronaries for Cardiogenic Shock. N Engl J Med. 1999;341:625-34.

7. Hochman JS, Sleeper LA, Godfrey E, et al. SHould we emergently revascularize Occluded Coronaries for cardiogenic shock: an international randomized trial of emergency PTCA/ CABG-trial design. The SHOCK Trial Study Group. Am Heart J. 1999;137:313-21.

8. Hochman JS, Buller CE, Sleeper LA, et al. Cardiogenic shock complicating acute myocardial infarction--etiologies, management and outcome: a report from the SHOCK Trial Registry. SHould we emergently revascularize Occluded Coronaries for cardiogenic shock? J Am Coll Cardiol. 2000;36(Suppl A):1063-70.

9. Hochman JS, Sleeper LA, White HD, et al. One-year survival following early revascularization for cardiogenic shock. JAMA. 2001;285:190-2.

10.Webb JG, Sanborn TA, Sleeper LA, et al. Percutaneous coronary intervention for cardiogenic shock in the SHOCK Trial Registry. Am Heart J. 2001;141:964-70.

11.Carnendran L, Abboud R, Sleeper LA, et al. Trends in cardiogenic 
shock: report from the SHOCK Study. The SHould we emergently revascularize Occluded Coronaries for cardiogenic shock? Eur Heart J. 2001;22:472-8.

12. Menon V, Fincke R. Cardiogenic shock: a summary of the randomized SHOCK trial. Congest Heart Fail. 2003;9:35-9.

13. Bangalore S, Gupta N, Guo Y, et al. Outcomes with invasive versus conservative management of cardiogenic shock complicating acute myocardial infarction. Am J Med. 2014;doi:10.1016/j. am.med.2014.12.009. [Epub ahead of print].

14.Dzavik V, Sleeper LA, Cocke TP, et al. SHOCK Investigators. Early revascularization is associated with improved survival in elderly patients with acute myocardial infarction complicated by cardiogenic shock: a report from the SHOCK Trial Registry. Eur Heart J. 2003;24:828-37.

15. ' Connor CM, Rogers JG. Evidence for overturning the guidelines in cardiogenic shock. N Engl J Med. 2012;367:1349-50.

16.Prondzinsky R, Lemm H, Swyter $M$, et al. Intra-aortic balloon counterpulsation in patients with acute myocardial infarction complicated by cardiogenic shock: the prospective, randomized IABP SHOCK Trial for attenuation of multiorgan dysfunction syndrome. Crit Care Med. 2010;38:152-60.

17. Thiele $H$, Zeymer $U$, Neumann FJ, et al. Intra-aortic balloon counterpulsation in acute myocardial infarction complicated by cardiogenic shock (IABP-SHOCK II): final 12 month results of a randomised, open-label trial. Lancet. 2013;382:1638-42.

18. Thiele $\mathrm{H}$, Zeymer $\mathrm{U}$, Neumann FJ, et al. Intraaortic balloon support for myocardial infarction with cardiogenic shock. N Engl J Med. 2012;367:1287-96.

19.Prodzinsky R, Unverzagt S, Russ M, et al. Hemodynamic effects of intra-aortic balloon counterpulsation in patients with acute myocardial infarction complicated by cardiogenic shock: the prospective, randomized IABP shock trial. Shock. 2012;37:37884.

20.Spiro J, Doshi SN. Use of left ventricular support devices during acute coronary syndrome and percutaneous coronary intervention. Curr Cardiol Rep. 2014;16:544.

21.Seyfarth M, Sibbing D, Bauer I, et al. A randomized clinical trial to evaluate the safety and efficacy of a percutaneous left ventricular assist device versus intra-aortic balloon pumping for treatment of cardiogenic shock caused by myocardial infarction. J Am Coll Cardiol. 2008;52:1584-8.

22. Moller-Helgestad OK, Poulsen CB, Christiansen EH, Lassen JF, Ravn HB. Support with intra-aortic balloon pump vs Impella 2.5 and blood flow to the heart, brain and kidneys - an experimental porcine model of ischaemic heart failure. Int J Cardiol. 2014;178C:153-8.

23.Jumean M, Pham DT, Kapur NK. Percutaneous bi-atrial extracorporeal membrane oxygenation for acute circulatory support in advanced heart failure. Catheter Cardiovasc Interv. 2014 dec 22. Doi:10.1002/ccd.25791. [Epub ahead of print].

24.Loforte A, Pilato E, Martin Suarez S, et al. RotaFlow and CentriMag Extracorporeal Membrane Oxygenation Support Systems as Treatment Strategies for Refractory Cardiogenic Shock. J Card Surg. 2014; doi:10.1111/jocs.12480. [Epub ahead of print].

25. TousekP, RokytaR, TesarovaJ, etal. Routineupfrontabciximabversus standard periprocedural therapy in patients undergoing primary percutaneous coronary intervention for cardiogenic shock: The PRAGUE-7 Study. An open randomized multicentre study. Acute Card Care. 2011;13:116-22.

26. Rokyta R, Pechman V, Tousek P, Pudil R, Lhotska J, Widimsky $P$. Routine pretreatment with abciximab versus standard periprocedural therapy in mechanically ventilated cardiogenic shock patients undergoing primary percutaneous coronary intervention: Subanalysis of the PRAGUE-7 study. Exp Clin Cardiol. 2013;18:81-4.

27.Alexander JH, Reynolds HR, Stebbins AL, et al. Effect of tilarginine acetate in patients with acute myocardial infarction and cardiogenic shock: the TRIUMPH randomized controlled trial. JAMA. 2007;297:1657-66.

28.Schillinger W, Hünlich M, Sossalla S, Hermann HP, Hasenfuss G. Intracoronary pyruvate in cardiogenic shock as an adjunctive therapy to catecholamines and intra-aortic balloon pump shows beneficial effects on haemodynamics. Clin Res Cardiol. 2011;100:433-8.

29. Husebye T, Eritsland J, Müller C, et al. Levosimendan in acute heart failure following primary percutaneous coronary intervention-treated acute ST-elevation myocardial infarction. Results from the LEAF trial: a randomized, placebo-controlled study. Eur J Heart Fail. 2013;15:565-72.

30.Dixon SR, Whitbourn RJ, Dae MW, et al. Induction of mild systemic hypothermia with endovascular cooling during primary percutaneous coronary intervention for acute myocardial infarction. J Am Coll Cardiol. 2002;40:1928-34.

31. Menon V, Webb JG, Hillis LD, et al. Outcome and profile of ventricular septal rupture with cardiogenic shock after myocardial infarction: a report from the SHOCK Trial Registry. SHould we emergently revascularize Occluded Coronaries in cardiogenic shock? J Am Coll Cardiol. 2000;36(Suppl A):1110-6.

32. Thompson CR, Buller CE, Sleeper LA, et al. Cardiogenic shock due to acute severe mitral regurgitation complicating acute myocardial infarction: a report from the SHOCK Trial Registry. SHould we use emergently revascularize Occluded Coronaries in cardiogenic shock? J Am Coll Cardiol. 2000;36(Suppl A):1104-9. 